\title{
Warum viele Spitzensportler unter Asthmabeschwerden leiden
}

— Überraschend häufig leiden Leistungssportler unter einer bronchialen Hyperreagibilität und Asthma, berichtete Prof. Dr. Winfried J. Randerath aus Solingen. So ergab eine Untersuchung bei Olympiateilnehmern eine Prävalenz von $8 \%$. Auffällig war, dass bestimmte Sportarten besonders häufig betroffen waren: Jeder vierte Triathlet litt unter asthmatischen Beschwerden, bei Schwimmern und Radrennfahrern lag die Prävalenz bei $17 \%$ und bei nordischen Skifahrern bei 15\% [Kippelen P et al. Br J Sports Med 2012; 46: 471-6]

Dies erkläre sich aus der Pathophysiologie des Anstrengungsasthmas, so Randerath. Die erhöhte körperliche Aktivität erfordert eine vermehrte Atmungsleistung und führt zu einer Steigerung der Ventilation. Dadurch kommt es zu einer Auskühlung und einem Feuchtigkeitsverlust in den Atemwegen, eine bronchiale Hyperreagibilität kann die
Folge sein. Gleichzeitig führt eine anhaltende Extrembelastung zu einer vermehrten Freisetzung von Entzündungsmediatoren und begünstigt chronisch-entzündliche Epithelschäden. Hochtrainierte Ausdauersportler sind daher besonders betroffen. Dabei wirkt sich auch das Umfeld aus, in dem der Sport betrieben wird: Kalte Temperaturen, Chloramine in Schwimmhallen sowie die Luftverschmutzung, beispielsweise beim Training an vielbefahrenen Straßen, erhöhen das Asthmarisiko.

Diagnostisch ist ein Anstrengungsasthma (",exercise-induced asthma“, EIA) von einer belastungsinduzierten Bronchokonstriktion ("exercise-induced bronchoconstriction", ElB) zu unterscheiden. Letztere tritt auf, ohne dass ein Asthma bronchiale zugrunde liegt und ist definiert als Abfall des forcierten exspiratorischen Volumens (FEV1) um 10\% in standardisierten Belastungstests. Differenzial-

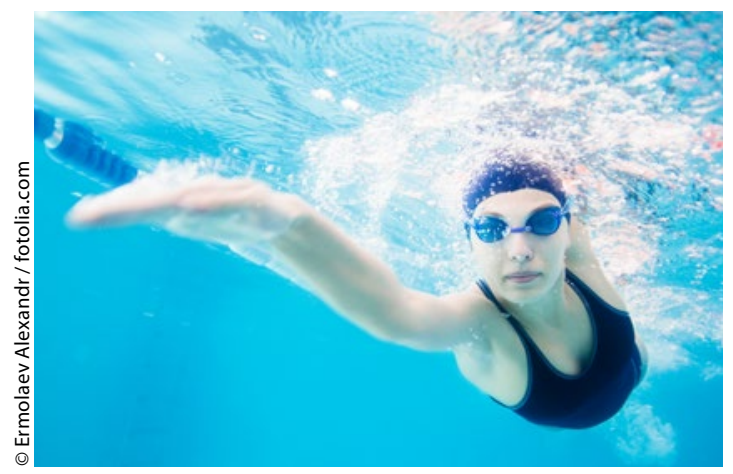

diagnostisch sei bei Luftnot im Training auch an einen Spasmus der Stimmbänder, eine "vocal cord dysfunction" (VCD) zu denken, so Randerath. Nicht selten sei aber Überanstrengung die Ursache der Atembeschwerden, zum Beispiel bei unzureichender körperlicher Fitness im Leistungssport oder auch bei übergewichtigen Kindern im Freizeitbereich.

Angelika Bauer-Delto

AllergoConnect: Asthma, Allergien und Sport

\section{Neues Positionspapier der GPA: Impfen macht nicht allergisch}

- "Spezifische Standard-Impfungen und Durchimpfung allgemein (höhere Anzahl insgesamt erhaltener Impfdosen) fördern nicht die allergische Sensibilisierung gegen Umweltallergene und fördern nicht die Entwicklung allergischer Erkrankungen wie Neurodermitis, Asthma und Heuschnupfen." Dies ist nur eines von 16 Statements, mit Hilfe derer die Gesellschaft für Pädiatrische Allergologie und Umweltmedizin (GPA) in

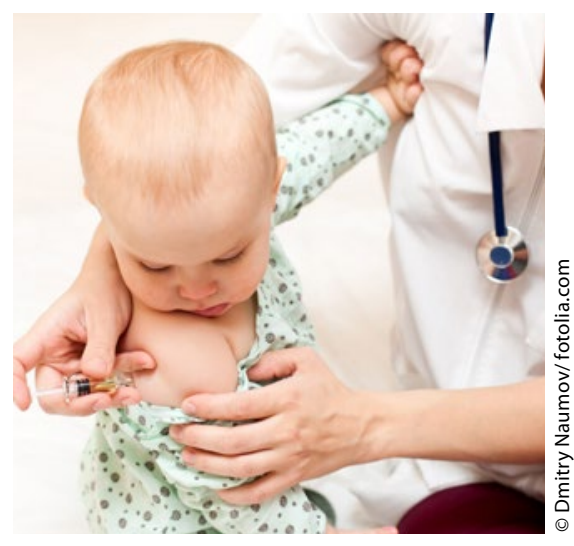

einem frisch aufgelegten Positionspapier den aktuellen Kenntnisstand über den Zusammenhang - oder besser gesagt NichtZusammenhang - von Impfen und Allergie auf den Punkt bringt.

Schaut man sich die Vielzahl der in Impfstoffen enthaltenen Allergene an, könnte man in Sachen Sensibilisierung in der Tat Schlimmes befürchten. Die Liste reicht über Fremdproteine, Antibiotika und Stabilisatoren bis hin zu Konservierungsmitteln, Impfantigenen und Adjuvanzien. Mit soliden Zahlen belegt werden konnten derartige Befürchtungen bislang jedoch nicht. Ganz im Gegenteil, wie die von PD Dr. Christoph Grüber, Frankfurt (Oder), vorgestellten Daten zeigen. Am Beispiel der schweren Neurodermitis konnte Grüber belegen, dass ein entsprechendes Risiko mit der kumulativen Zahl der Impfdosen sogar sinkt. Auch für die sich zum Teil hartnäckig haltende Vermutung, dass durchgemachte Infektionen das Allergierisiko senken können, gibt es nach Darstellung Grübers keine Belege - auch nicht im Zusammenhang mit
Pertussis und Masern. Während Masernund Keuchhustenimpfung keinerlei Effekte auf atopische Manifestationen hatten, waren durchgemachte Infektionen hier und da sogar mit einem erhöhten Allergierisiko assoziiert, berichtete der maßgeblich an der Erarbeitung des Positionspapiers beteiligte Kinderarzt.

Mit dem neuen Positionspapier bekommen Ärzte auch konkrete Informationen an die Hand, wie mit Kindern zu verfahren ist, die ein deutlich erhöhtes Allergierisiko haben oder bei denen es in der Vorgeschichte im Rahmen einer Impfung zu einer allergischen Sofortreaktion gekommen ist. In solchen Fällen kann es ratsam sein, nachfolgende Impfungen im stationären Umfeld, mit halbierter Impfdosis und mit einer entsprechend langen Nachbeobachtungszeit vorzunehmen. Nach einer allergischen Impfreaktion spricht sich das Positionspapier grundsätzlich für die Durchführung von Hauttests mit dem Impfstoff beziehungsweise Einzelkomponenten des Impfstoffs aus, um das Risiko zukünftiger Impfstoffreaktionen zu minimieren.

Dr. Ludger Riem

AllergoConnect: Allergie und Impfen 